Revisión de la literatura 


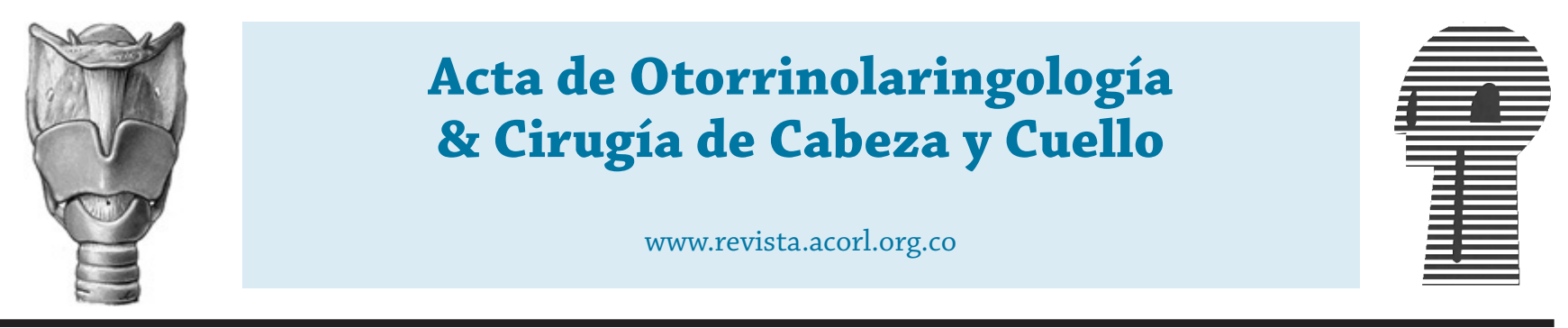

Revisión de la literatura

\section{Proceso de decanulación electiva en pacientes con traqueotomía: búsqueda de criterio}

\section{Elective decannulation process in tracheostomy patients: criterion search}

Juan Antonio Lugo-Machado*, Martha Janeth Jiménez-Rodríguez**.

* Médico otorrinolaringólogo; profesor de posgrado del Hospital de Especialidades N. 2 "Lic. Luis Donaldo Colosio Murrieta", Instituto Mexicano del Seguro Social; profesor de la Escuela de Medicina de la Universidad de Sonora, Campus Cajeme. Ciudad Obregón, Sonora. ORCID: https://orcid.org/0000-0003-4864-8546

** Médico residente de otorrinolaringología del Hospital de Especialidades N. "Lic. Luis Donaldo Colosio Murrieta", Instituto Mexicano del Seguro Social. Ciudad Obregón, Sonora. ORCID: https://orcid.org/0000-0002-0923-6111

Forma de citar: Lugo-Machado JA, Jiménez-Rodríguez MJ. Proceso de decanulación electiva en pacientes con traqueotomía: búsqueda de criterio. Acta otorrinolaringol. cir. cabeza cuello. 2021;49(4): 310-314 DOI.10.37076/acorl.v49i4.502

\section{INFORMACIÓN DEL ARTÍCULO}

Historia del artículo:

Recibido: 2 de mayo de 2020

Evaluado: 18 de noviembre de 2021

Aceptado: 20 de noviembre de 2021

Palabras clave (DeCS):

Cánula, traqueotomía, selección de paciente, retiro.

\section{RESUMEN}

Los progresos en la ciencia y la tecnología en el ámbito de la salud y, en concreto, en la unidad de cuidados intensivos (UCI) hospitalarios han incrementado la supervivencia en la población que demanda atención médica; no obstante, también han generado una población que requiere cuidados y manejos específicos, en su mayoría de manera multidisciplinaria, entre ellos, los pacientes que requieren de atención a traqueotomía. La necesidad de estandarizar el proceso de decanulación es una carencia no cubierta. En esta revisión narrativa exponemos algunos criterios, protocolos o guías vertidas por los autores consultados, sin que hasta el momento exista una guía estandarizada. 


\section{ABSTRACT}

Key words (MeSH):

Cannula; Tracheotomy; Selection, Patient; retirement.
Progress in science and technology in the health field, and specifically in the hospital intensive care unit, has increased survival in the population that requires medical care; however, it has also generated a population that requires specific care and management, mostly in a multidisciplinary way, including patients who require attention to a tracheostomy. The need to standardize the decannulation process is an unmet deficiency. In this narrative review, we expose some criteria, protocols or guidelines issued by the authors consulted, so far there is no standardized guide.good surgical and clinical results in the vast majority of cases.

\section{Introducción}

A lo largo de las décadas, el uso de apoyo ventilatorio ha reducido la mortalidad de pacientes críticos, resultando en la conversión de muchas condiciones letales a pacientes con secuelas residuales que requieren de manejo específicos (1). La traqueotomía es un procedimiento frecuente en pacientes que requieren ventilación mecánica prolongada y protección de las vías respiratorias en la unidad de cuidados intensivos (UCI) (2). Cada año, alrededor de 800000 residentes de los Estados Unidos se someten a ventilación mecánica debido a una insuficiencia respiratoria aguda $(3,4)$. Algunos estudios han documentado un aumento significativo en el número de pacientes que requieren ventilación mecánica $(5,6)$.

Estadísticas en los Estados Unidos, concretamente en Carolina del Norte, han descrito un aumento del $11 \%$ en la ventilación mecánica para adultos, de 284/100 000 en 1996 a 314/100 000 en 2002, con un incremento en el grupo de edad en jóvenes (un $19 \%$ para los de edad entre 18 a 64 años) (7). Se señala que hasta el $34 \%$ de los pacientes que necesitan ventilación mecánica durante $>48$ horas reciben una traqueotomía (8). Datos en la Unión Americana señalan que en 1996 se colocaron alrededor de 64000 cánulas de traqueotomía (9).

Los efectos de la cánula de traqueotomía en pacientes sin ventilación mecánica generan cambios en la biomecánica de la deglución, entre los que se incluyen la reducción de la elevación laríngea, la insuficiencia en el cierre glótico y la dificultad del paso del bolo debido al globo externo; asimismo, se generan alteraciones en la sensibilidad para el reflejo de la tos $(10,11)$.

Los cambios en la mecánica de la deglución resultantes no solo están relacionados con un mayor riesgo de aspiración, sino que también se generan cambios en la fase faríngea de la deglución $(12,13)$. La incapacidad para hablar con el tubo de traqueotomía in situ produce ansiedad, frustración y depresión significativas entre los pacientes (14).

El proceso de retiro de la cánula de traqueotomía y el mantenimiento de la respiración espontánea con la protección de las vías aéreas se denomina decanulación. Este paso aparentemente simple requiere una coordinación casi perfecta de los mecanismos regulatorios neurológicos, la deglución, la tos, la fonación y los músculos respiratorios (15).

\section{Desarrollo}

La decanulación exitosa se define como el retiro de la cánula y el cierre del sitio sin complicaciones respiratorias consecuentes o un deterioro de los gases en sangre durante al menos dos semanas. El fracaso se define por la aparición de dificultad respiratoria, la disminución de la capacidad vital y la saturación de oxihemoglobina a pesar del uso de presión positiva (16). Existen una serie de factores que podrían incrementar el riesgo de aspiración inmediatamente después de la decanulación: la edad avanzada, la obesidad, el mal estado neurológico, la sepsis y el mal manejo de secreciones son algunas de las razones principales de la decanulación fallida (17). Varios estudios han enfatizado la importancia de la decanulación dentro de la UCI o estancia hospitalaria debido a una mejor atención, en comparación con la ambulatoria $(18,19)$; no obstante, en hospitales de países con escasos recursos esto representaría costos elevados de estancia hospitalaria. A pesar de lo relevante e importante de la decanulación, no existe un protocolo universalmente aceptado. Existe una diversidad en los criterios, protocolos y guías para el proceso de retiro de una cánula $(20,21)$.

En la mayoría de los casos, estas evaluaciones se basan en la impresión clínica subjetiva del médico, que tiene una base puramente empírica (22). Existen pocos criterios reconocidos y globalmente aceptados para guiar los procesos del retiro de cánula, ya que las publicaciones son reducidas al respecto. Una decanulación prematura o insegura aumenta los riesgos y la morbilidad en el paciente. Es imperativo que los criterios de decanulación se evalúen de manera sólida para garantizar un manejo con el menor de los riesgos. En su escrito, McDonald y colaboradores señalan que cuatro de sus criterios de decanulación parecen sólidos para apoyar su retiro de una manera segura (23). Cohen y colaboradores confirman que una evaluación endoscópica con movilidad normal de las cuerdas vocales y la supraglotis, antes de la decanulación, es la alternativa más segura y rápida en comparación con la técnica tradicional (24). Graves y colaboradores también reiteraron lo importante de la fibroscopia de la vía aérea para alcanzar una buena tasa de éxito (25). Del mismo modo, la evaluación subjetiva de la tos normal es habitual; Bach y colaboradores, Ceriana y colaboradores, Chan y colaboradores, y Guerlain y colaboradores utilizaron 
una medida objetiva de una tos efectiva para decidir sobre el retiro de la cánula $(16,20,26,27)$; sin embargo, el método adoptado de decanulación es considerablemente variable. Mientras que algunos autores prefirieron la oclusión del tubo traqueal después de reducir el tamaño de tubo, otros ocluyen inmediatamente el tubo sin reducir el tamaño, y distintos autores eliminaron abruptamente el tubo traqueal $(21,28,29)$.

La elección del método se basa en la tolerabilidad del procedimiento de oclusión por parte del paciente y también en la experiencia del médico. No existe un método universalmente aceptado. Además, también existe discrepancia en el período de observación antes del retiro para considerarla exitosa. La falta o ausencia de un estudio controlado aleatorio en este aspecto es un obstáculo importante para estandarizar algún protocolo o guía de decanulación. Singh y colaboradores señalan una lista de verificación que incluye la evaluación en la integridad del estado de alerta, las características de las secreciones, necesidad y frecuencia de aspiración, efectividad en la deglución, el reflejo tusígeno y la permeabilidad de las vías respiratorias (22). El paciente debe estar consciente, orientado y ser capaz de mantener una vía aérea permeable. Las secreciones deben ser fáciles de manejar por el paciente y la frecuencia de succión debe ser inferior a 4 en las 24 horas anteriores. El paciente debe ser capaz de tragar líquidos/ semisólidos sin riesgo de aspiración y tener una tos adecuada con una buena tasa de flujo espiratorio máxima (22). Lanini y colaboradores indican que, en pacientes con lesión cerebral severa, la fibroscopia laríngea conduce al retiro exitoso de la cánula, incluso en una proporción relevante de los pacientes que no cumplen los criterios de decanulación con una base puramente clínica (30).

En la actualidad, la evidencia aún es muy limitada y requiere más investigaciones, concretamente con la validación de los diferentes parámetros utilizados. Para Kutsukutsa y colaboradores, un enfoque de protocolo para el retiro de la cánula puede ser inapropiado y señala en cambio un enfoque algorítmico que utiliza parámetros validados (31). El manejo multidisciplinario con la interacción entre médicos, enfermeras, fisioterapeutas y logopedas permite una reducción en el tiempo de uso de la traqueotomía, acelera el proceso de su retiro y lo hace más seguro para los pacientes. Mendes y colaboradores sugieren que un diagrama de flujo con participación interdisciplinaria puede hacer que el proceso de retiro de la cánula sea más seguro, minimizando el riesgo de complicaciones (32). Welton, Mah y Madeiros señalan que entre los miembros que forman parte de este equipo multidisciplinario, la literatura destaca la participación de médicos, fisioterapeutas, logopedas y enfermeras, los cuales están involucrados en una etapa diferente del proceso de decanulación (33-35).

Recapitulando, podemos indicar que los factores identificados como indicativos de éxito en el proceso de decanulación son la estabilidad clínica y hemodinámica, el nivel de alerta y la colaboración del paciente, sin necesidad de ventilación mecánica ni dependencia de la humidificación, buen manejo de las secreciones y ausencia de aspiración; dentro de los pasos más importantes en el proceso de decanulación se destaca desinflar el globo, ver la permeabilidad de la vía respiratoria superior, la evaluación de la mecánica de la deglución, el manejo de la secreción y el entrenamiento de oclusión de traqueotomía.

\section{Conclusiones}

Es claro que existen áreas de oportunidad en la creación de un protocolo estandarizado que nos facilitan realizar una decanulación de pacientes traqueotomizados con el mayor índice de éxito y menor probabilidad de fracaso. La ausencia de estudios controlados aleatorizados, que apoyen la creación de una serie de criterios para la decanulación universalmente aceptados, obstaculiza el avance en este sector que nos apremia. Dentro de los métodos para lograr el retiro de la cánula, algunos autores señalan el cambio de cánula de forma gradual a una de menor tamaño para posteriormente realizar la oclusión de la misma, y que al tener una tolerancia de 12 horas o más se tiene un mayor porcentaje de éxito para su retiro. Gran número de autores emplean diversos criterios elaborados de forma empírica y otros señalan una lista de verificación que considera los siguientes puntos: integridad del estado de alerta, características de la secreción y la frecuencia de aspiración, mecánica de la deglución efectiva, reflejo de tos conservado y efectivo, y permeabilidad de la vía aérea. El empleo de la endoscopia de la vía aérea superior se destaca por unos autores como un procedimiento indispensable para lograr una decanulación exitosa. Para finalizar, es necesario hacer énfasis en lo que resaltan algunos autores en relación con que el proceso de retiro de cánula requiere de un manejo multidisciplinario.

\section{Fuentes de financiamiento}

Este artículo ha sido financiado por los autores.

\section{Aspectos éticos}

Se trata de un escrito de revisión narrativa en el que no se realizan experimentos en seres humanos ni emplea datos sensibles de los expedientes clínicos, por lo que es considerado sin riesgo para las personas.

\section{Agradecimientos}

Agradecemos a nuestros compañeros médicos residentes, médicos internos, enfermeras y distintos profesionales de la salud en formación que nos brindaron su apoyo durante la estructuración y redacción del manuscrito.

\section{Limitaciones del estudio}

Se trata de una revisión narrativa con limitaciones relacionadas a la opinión personal de los autores. 


\section{REFERENCIAS}

1. Kress JP, Pohlman AS, O’Connor MF, Hall JB. Daily interruption of sedative infusions in critically ill patients undergoing mechanical ventilation. N Engl J Med. 2000;342(20):1471-7. doi: 10.1056/NEJM200005183422002

2. Esteban A, Anzueto A, Alía I, Gordo F, Apezteguía C, Pálizas $\mathrm{F}$, et al. How is mechanical ventilation employed in the intensive care unit? An international utilization review. Am J Respir Crit Care Med. 2000;161(5):1450-8. doi: 10.1164/ ajrccm.161.5.9902018

3. Cheung NH, Napolitano LM. Tracheostomy: Epidemiology, indications, timing, technique, and outcomes. Respiratory Care. 2014;59(6):895-19. doi: 10.4187/respcare.02971

4. Wunsch H, Linde-Zwirble WT, Angus DC, Hartman ME, Milbrandt EB, Kahn JM. The epidemiology of mechanical ventilation use in the United States. Crit Care Med. 2010;38(10):1947-53. doi: 10.1097/CCM.0b013e3181ef4460

5. Needham DM, Bronskill SE, Sibbald WJ, Pronovost PJ, Laupacis A. Mechanical ventilation in Ontario, 1992-2000: incidence, survival, and hospital bed utilization of noncardiac surgery adult patients. Crit Care Med. 2004;32(7):1504-9. doi: 10.1097/01.ccm.0000129972.31533.37

6. Needham DM, Bronskill SE, Calinawan JR, Sibbald WJ, Pronovost PJ, Laupacis A. Projected incidence of mechanical ventilation in Ontario to 2026: Preparing for the aging baby boomers. Crit Care Med. 2005;33(3):574-9. doi: 10.1097/01. ccm.0000155992.21174.31

7. Carson SS, Cox CE, Holmes GM, Howard A, Carey TS. The changing epidemiology of mechanical ventilation: a population-based study. J Intensive Care Med. 2006;21(3):17382. doi: $10.1177 / 0885066605282784$

8. Quality of Life After Mechanized Ventilation in the Elderly Study Investigators. 2-month mortality and functional status of critically ill adult patients receiving prolonged mechanical ventilation. Chest. 2002;121(2):549-58. doi: 10.1378/ chest.121.2.549

9. Owings MF, Kozak LJ. Ambulatory and inpatient procedures in the United States, 1996. Vital Health Stat 13. 1998;(139):1-119.

10. Everitt E. Managing the weaning of a temporary tracheostomy. Nurs Times. 2016;112(20):17-9.

11. Pryor L, Ward E, Cornwell P, O'Connor S, Chapman M. Patterns of return to oral intake and decannulation post-tracheostomy across clinical populations in an acute inpatient setting. Int $\mathrm{J}$ Lang Commun Disord. 2016;51(5):556-67. doi: 10.1111/14606984.12231

12. Zanata IL, Santos RS, Marques JM, Hirata GC, Santos DA. Speech-language pathology assessment for tracheal decannulation in patients suffering from traumatic brain injury. Codas. 2016;28(6):710-16. Portuguese, English. doi: 10.1590/2317-1782/20162014086

13. Barros APB, Portas JG, Queija D dos S. Implicações da traqueostomia na comunicação e na deglutição: [revisão] / Tracheotomy implication upon communication and swallowing: [review]. Rev. bras. cir. cabeça pescoço. 2009;38(3):202-7.

14. O'Connor HH, Kirby KJ, Terrin N, Hill NS, White AC. Decannulation following tracheostomy for prolonged mechanical ventilation. J Intensive Care Med. 2009;24(3):18794. doi: 10.1177/0885066609332701

15. Garuti G, Reverberi C, Briganti A, Massobrio M, Lombardi F, Lusuardi M. Swallowing disorders in tracheostomised patients: a multidisciplinary/multiprofessional approach in decannulation protocols. Multidiscip Respir Med. 2014;9(1):36. doi: 10.1186/2049-6958-9-36
16. Bach JR, Saporito LR. Criteria for extubation and tracheostomy tube removal for patients with ventilatory failure. A different approach to weaning. Chest. 1996;110(6):1566-71. doi: 10.1378/chest.110.6.1566

17. Schmidt U, Hess D, Bittner E. To decannulate or not to decannulate: a combination of readiness for the floor and floor readiness? Crit Care Med. 2011;39(10):2360-1. doi: 10.1097/ CCM.0b013e318226618a

18. Martinez GH, Fernandez R, Casado MS, Cuena R, Lopez-Reina $\mathrm{P}$, Zamora S, et al. Tracheostomy tube in place at intensive care unit discharge is associated with increased ward mortality. Respir Care. 2009;54(12):1644-52.

19. Fernandez R, Bacelar N, Hernandez G, Tubau I, Baigorri F, Gili $\mathrm{G}$, et al. Ward mortality in patients discharged from the ICU with tracheostomy may depend on patient's vulnerability. Intensive Care Med. 2008;34(10):1878-82. doi: 10.1007/ s00134-008-1169-6

20. Ceriana P, Carlucci A, Navalesi P, Rampulla C, Delmastro $\mathrm{M}$, Piaggi $\mathrm{G}$, et al. Weaning from tracheotomy in long-term mechanically ventilated patients: feasibility of a decisional flowchart and clinical outcome. Intensive Care Med. 2003;29(5):845-8. doi: 10.1007/s00134-003-1689-Z

21. Shrestha KK, Mohindra S, Mohindra S. How to decannulate tracheostomised severe head trauma patients: a comparison of gradual vs abrupt technique. Nepal Med Coll J. 2012;14(3):20711.

22. Singh RK, Saran S, Baronia AK. The practice of tracheostomy decannulation-a systematic review. J Intensive Care. 2017;5:38. doi: 10.1186/s40560-017-0234-z

23. McDonald H, Thomas AJ. Outcome of physiotherapy led decannulation from tracheostomy practice in a large London teaching hospital. Physiotherapy. 2015;101(1):e1510-e1511. doi: 10.1016/j.physio.2015.03.1493

24. Cohen O, Tzelnick S, Lahav Y, Stavi D, Shoffel-Havakuk $\mathrm{H}$, Hain M, et al. Feasibility of a single-stage tracheostomy decannulation protocol with endoscopy in adult patients. Laryngoscope. 2016;126(9):2057-62. doi: 10.1002/lary.25800

25. Rumbak MJ, Graves AE, Scott MP, Sporn GK, Walsh FW, Anderson WM, et al. Tracheostomy tube occlusion protocol predicts significant tracheal obstruction to air flow in patients requiring prolonged mechanical ventilation. Crit Care Med. 1997;25(3):413-7. doi: 10.1097/00003246-199703000-00007

26. Chan LY, Jones AY, Chung RC, Hung KN. Peak flow rate during induced cough: a predictor of successful decannulation of a tracheotomy tube in neurosurgical patients. Am J Crit Care. 2010;19(3):278-84. doi: 10.4037/ajcc2009575

27. Guerlain J, Guerrero JA, Baujat B, St Guily JL, Périé S. Peak inspiratory flow is a simple means of predicting decannulation success following head and neck cancer surgery: a prospective study of fifty-six patients. Laryngoscope. 2015;125(2):365-70. doi: 10.1002/lary.24904

28. Choate K, Barbetti J, Currey J. Tracheostomy decannulation failure rate following critical illness: a prospective descriptive study. Aust Crit Care. 2009;22(1):8-15. doi: 10.1016/j. aucc.2008.10.002

29. Tobin AE, Santamaria JD. An intensivist-led tracheostomy review team is associated with shorter decannulation time and length of stay: a prospective cohort study. Crit Care. 2008;12(2):R48. doi: 10.1186/cc6864

30. Lanini B, Binazzi B, Romagnoli I, Chellini E, Pianigiani L, Tofani A, et al. Tracheostomy decannulation in severe acquired brain injury patients: The role of flexible bronchoscopy. Pulmonology. 2021:S2531-0437(21)00115-X. doi: 10.1016/j. pulmoe.2021.05.006 
31. Kutsukutsa J, Kuupiel D, Monori-Kiss A, Del Rey-Puech P, Mashamba-Thompson TP. Tracheostomy decannulation methods and procedures for assessing readiness for decannulation in adults: a systematic scoping review. Int J Evid Based Healthc. 2019;17(2):74-91 . doi: 10.1097/ XEB.0000000000000166

32. Mendes TAB, Cavalheiro LV, Arevalo RT, Sonegth R. Preliminary study on a proposal of an interdisciplinary flowchart of tracheostomy decannulation. Einstein. 2008;6(1):1-6.

33. Medeiros GC de, Sassi FC, Lirani-Silva C, Andrade CRF de. Critérios para decanulação da traqueostomia: revisão de literatura. CoDAS. 2019;31(6):e20180228. doi: 10.1590/2317$1782 / 20192018228$

34. Welton C, Morrison M, Catalig M, Chris J, Pataki J. Can an interprofessional tracheostomy team improve weaning to decannulation times? A quality improvement evaluation. Can J Respir Ther. 2016;52(1):7-11.

35. Mah JW, Staff II, Fisher SR, Butler KL. Improving Decannulation and Swallowing Function: A Comprehensive, Multidisciplinary Approach to Post-Tracheostomy Care. Respir Care. 2017;62(2):137-43. doi: 10.4187/respcare.04878 